\title{
PERFORMANCE OF OPTICAL BURST SWITCHED WDM RING NETWORK WITH TTFR SYSTEM
}

\author{
Yutaka Arakawa, Naoaki Yamanaka, and Iwao Sasase \\ Department of Information and Computer Science, Keio University, Japan
}

\begin{abstract}
:
In this paper, we propose an architecture of Optical Burst Switched WDM ring network. In our proposed OBS ring network, every node is equipped with one tunable transmitter and one fixed-tuned receiver (TTFR) oparating on a given wavelength that identifies the node. TTFR type ring network has an advantage that no receiver collisions occur and each node can detect channel collision by transmitter side. By computer simulations, we evaluate the performance of throughput, goodput, and queueing delay. As a result, we show the performance effectiveness of our proposed architecture and access protocol.
\end{abstract}

\section{INTRODUCTION}

Optical burst switching (OBS) [1]- [2] is a switching technique that occupies the middle of the spectrum between the well-known circuit switching and packet switching paradigms. In OBS network, several IP packets with the same destinations are assembled into a burst, and forwarded through the network in optical domain. The transmission of each burst is proceeded by the transmission of a burst header packet, which usually takes place on a separate single channel. Recently, as an alternative of SONET/SDH based metro ring network, WDM metro ring network is focused on. SONET/SDH network represents a significant investment on the part of carries, and are currently being upgraded to support WDM. A research on the WDM metro ring network with optical packet switching, such as HORNET (Hybrid Optoelectronic Ring NETwork) [3], attracts much attention. On the other hand, the research on the WDM metro ring network with optical burst switching is also done [4]. In [4], OBS metro ring network consists of $N$ nodes, and each node owns a home wavelength on which it transmits its bursts. The ring operates under the fixed tuned transmitter and tunable receiver (FTTR) system. In this system, every node 
can transmit without worrying about channel collisions since no other node can transmit with the same wavelength. However, it is possible that several packets arrive at the same node simultaneously. At that time all the bursts except one, that is randomly selected, are discarded. In this way, FTTR type ring network waste a bandwidth by the reason that bursts that might be discarded are transmitted to the destination.

In this paper, we propose an architecture of Optical Burst Switched WDM ring network. In our proposed OBS ring network, every node is equipped with one tunable transmitter and one fixed-tuned receiver (TTFR), and transmits bursts on the wavelength assigned for each destination. Since all nodes use the same wavelength for transmitting a packet to the same destination, channel collisions occur in the case that multiple nodes transmits bursts to a same destination node. However, TTFR has an advantage that causes no receiver collisions. In addition, since a burst header packet is transmitted ahead of a data burst under JET (Just Enough Time) signaling, channel collisions are detected in advance. A node that detects a channel collisions stops transmission of the burst immediately, and retransmits the burst after going through a burst of upstream node. By such upstream prioritized switching, bursts transmitted successfully will be received certainly at the destination node without being discarded during a transfer. By computer simulations, we evaluate the performance of throughput, goodput, and queueing delay. As a result, we show the performance effectiveness of our proposed architecture and access protocol.

The rest of the paper is organized as follows. We present our proposed architecture and access protocol in Section 2. The performance evaluations are shown in Section 3. Finally, we discuss the obtained results.

\section{PROPOSED OBS RING NETWORK}

\subsection{Ring and node architecture}

We consider $N$ nodes organized in a unidirectional ring, as shown in Fig. 1. The ring can be a metropolitan area network (MAN) serving as the backbone that interconnects a number of access networks, and transporting multiple types of traffic from users, such as IP traffic, ATM traffic, frame relay traffic. Each fiber link between two consecutive OBS nodes in the ring can support $N+1$ wavelengths. Of these, $N$ wavelengths are used to transmit bursts, and $(N+1)$ th wavelength is used as the control channel.

Each OBS node is attached to one or more access networks. In the direction from the access networks to the ring, the OBS node acts as concentrator. It collects and buffers electronically data, transmitted by users over the access networks, which need to be transported over the ring. Buffered data are subsequently grouped together and transmitted in a burst to the destination OBS node. A burst can be of any size between a minimum and maximum value. 


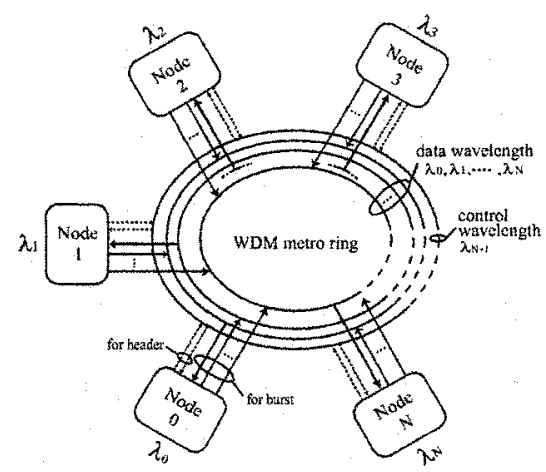

Figure 1. An architecture of Optical Burst Switched WDM ring network

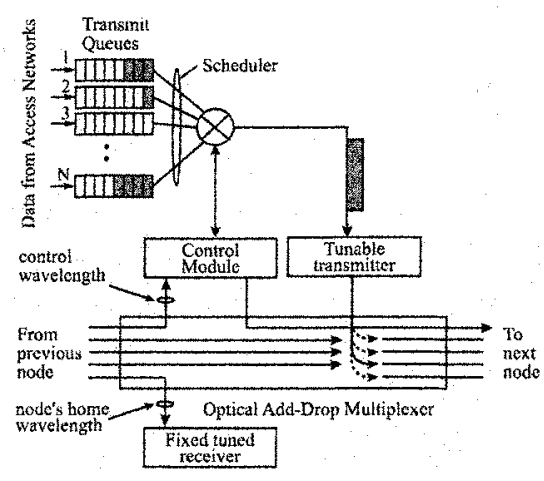

Figure 2. An architecture and operation of every node

Bursts travel as optical signals along the ring, without undergoing any electrooptic conversion at intermediate nodes. In the other direction from the ring to the access networks, an OBS node terminates optical bursts destined to it, electronically processes the data contained there in, and delivers them to users in its attached access networks.

The architecture of an OBS node is shown in Fig. 2. Each node is equipped with one optical add-drop multiplexer (OADM), and two pairs of optical trans ceivers. The first pair consists of a receiver and transmitter fixed tuned to the control wavelength, and are part of the control module in Fig. 2. The control wavelength is dropped by the OADM at each node, and added back after the control module has read the control information and has inserted new information. The second pair of transceivers consists of a tunable transmitter and a receiver that is tuned to the node's home wavelength. The OADM drops the optical signal on the node's home wavelength. In OBS network, several IP packets with the same destinations are assembled into a burst, and forwarded through the network in optical domain. The transmission of each burst is proceeded by the transmission of a burst header packet, which usually takes place on a separate single channel. We use Just Enough Time (JET) proposed in [1] as the signaling protocol. It starts transmitting the data burst soon after the transmission of the burst header packet. We will refer to the interval of time between the transmission of the first bit of the burst header packet and the transmission of the first bit of the data burst as the offset. The burst header packet carries information about the burst, including the offset value, the length of the burst, its priority, etc.. The purpose of burst header packets is to inform each intermediate node of the upcoming data burst, so that it can configure its switch fabric appropriately to switch the burst to the appropriate output port. 


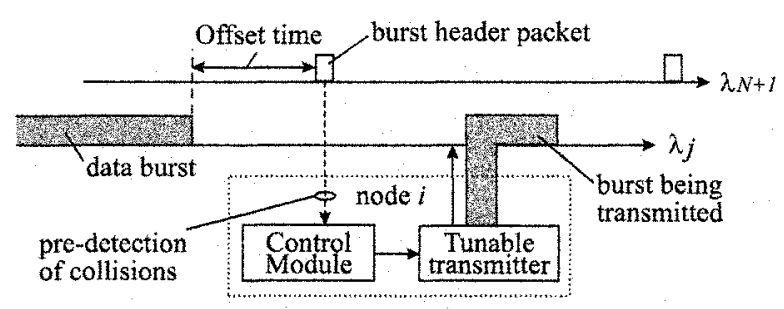

Figure $3 a$. Incoming burst from upstream node is detected

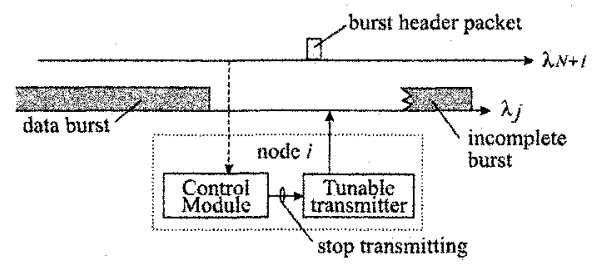

Figure $3 b$. Example of upstream prioritized switching

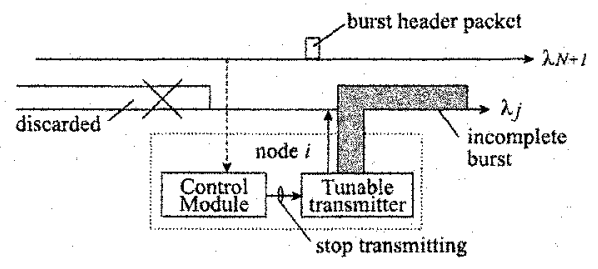

Figure 3c. Example of earliest arrival prioritized switching

\subsection{Access protocol}

In the proposed OBS ring network, channel collisions occur in the case of transmitting bursts to a given destination node from multiple nodes, since all nodes will transmit on the same wavelength of the destination. Therefore, an access protocol in consideration of channel collisions is required. A basic access protocol is similar to CSMA/CD (Carrier Sense Multiple Access with Collision Detection). Since a burst header packet that includes the information of the burst, such as length and destination, is transmitted ahead of a data burst, nodes can detect channel collisions in advance. Fig. 3a shows a basic access protocol with Collision Detection. When a node $i$ transmits a packet towards node $j$, it confirms the availability of wavelength $\lambda_{j}$ for a term of transmitting. If a node can transmit, a control packet is transmitted immediately on control wavelength, and after a delay of offset time, a payload is transmitted. We can classify access protocols into Upstream Prioritized Switching or Earliest Arrival Prioritized Switching according to the operation at channel collisions. Fig. 3b shows the example of upstream prioritized switching. The node which has detected a channel collision by the control packet stops transmission of the burst immediately. By upstream prioritized switching, bursts transmitted successfully at the source node will be received certainly at the destination node without being discarded during a transfer. Fig. $3 \mathrm{c}$ shows the example of earliest arrival prioritized switching. In the case that node $i$ has already started to transmit a given burst, the node which has detected a channel collision will drop the burst from upstream node. By earliest arrival prioritized switching, 
although bursts may be discarded by the relay node, it can prevent imcomplete bursts due to the transmit interruption being transmitted downstream.

\subsection{Burst Assembly}

Data from access network is organized into transmit queues according to their destination. The data buffer at each OBS node is shared by $N-1$ transmit queues, each corresponding to one of the $N-1$ destination nodes. The order in which transmit queues are served is determined by the scheduler in Fig. 2. A transmit queue is available for service if its size is larger than Min Burst Size, or the first data of the transmit queue has waited for more than timeout time. If the size of available transmit queue is less than Max Burst Size, then a burst that includes all data in the transmit queue is constructed. Otherwise, a burst of at most size Max Burst Size is constructed, and the data remaining in the transmit queue is served at a later time. If two or more transmit queues are available, we choose one queue in round-robin or random manner. Although round-robin can keep the fairness for every destinaion, the synchronization of collision may occur between adjacent nodes. When channel collision occurs by the reason that a node $i$ and a node $i+1$ transmit bursts to the same destination $j$, to the following timing, it's likely that they subsequently transmit a burst to the same destination, such as node $j+1$. Therefore, in order to improve the synchronization problem, we propose a random queue selection.

\section{PERFORMANCE EVALUATION}

\subsection{Simulation model}

In our simulation study we consider a ring network with 6 nodes, each with an electronic buffer of $10 \mathrm{MB}$. The distance between two successive nodes in the ring is taken to be $4 \mathrm{~km}$. We assume that the control wavelength each burst wavelength runs at $2.5 \mathrm{Gbps}$. We assume that data arrives in packets, and the packet arrival process to each node is described by a modified interrupted poisson process (IPP) [5]. This modified IPP is an ON/OFF process, where both the ON and the OFF periods are exponentially distributed. Packets arrive back to back during the ON period at the rate of $A_{\text {on }}=2.5$ Gbps. No packets arrive during the OFF period. The packet size is assumed to follow a truncated exponential distribution with an average size of 500 bytes and a maximum size of 5000 bytes. We use the squared coefficient of variation, $c^{2}$ and of the packet inter arrival time to measure the burstiness of the arrival process. $c^{2}$ is defined as the ratio of the variance of the packet inter arrival time divided by the squared mean of the packet inter arrival time. We use the expression for the $c^{2}$ of an IPP and where the packet size is not truncated. We have $c_{I P P}^{2}=$ $1+\frac{2 \lambda \mu_{1}}{\left(\mu_{1}+\mu_{2}\right)^{2}}$ where $1 / \lambda=(500$ bytes $) /(2.5 \mathrm{Gbps})=1.6 \mu \mathrm{s}$, and $1 / \mu_{1}$ and $1 / \mu_{2}$ 


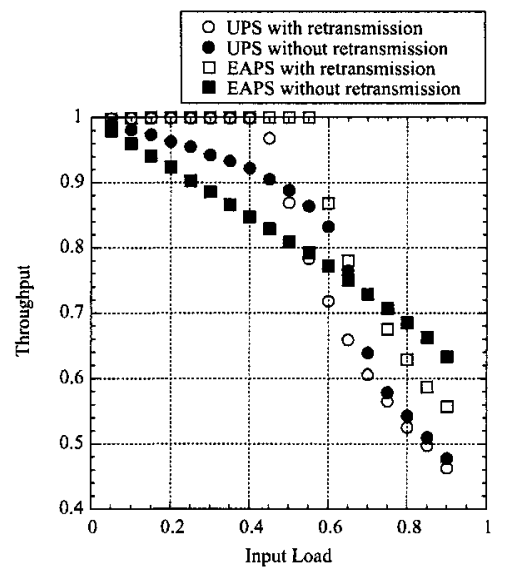

Figure 4. Comparison of UPS and EAPS about throughput performance

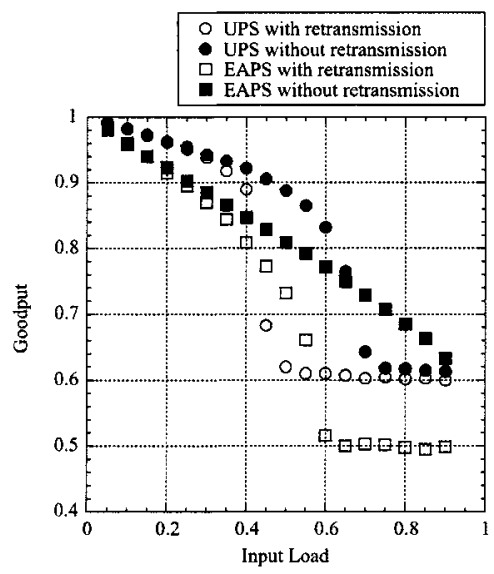

Figure 5. Comparison of UPS and EAPS about goodput performance

are the mean times of the $\mathrm{ON}$ and OFF periods, respectively. To completely characterize the arrival process, we use the above expression for $c^{2}$ and another equation that involves the mean times of the $\mathrm{ON}$ and OFF periods. We define the quantity $A_{r}=A_{o n} \frac{\mu_{2}}{\mu_{1}+\mu_{2}}$ Given the $c^{2}$ and the average packet arrival rate $A_{r}$, we can calculate the quantities $\mu_{1}$ and $\mu_{2}$, and therefore the arrival process is completely characterized.

In this section, we evaluate throughput, goodput, delay by a computer simulation. These performance measures are estimated by varying an input load from 0.1 to 1.0 with an increment of 0.05 . In this paper, an input load shows the average packet arriving rate into a single node. The destination of packets follows the uniform distribution. $c^{2}$ of packet inter-arrival time at each node is set to 20. We also set Max Burst Size to $50 \mathrm{~KB}$, TimeOut to $4 \mathrm{~ms}$ and offset value to $10 \mu \mathrm{s}$. In each figure, UPS and EAPS represent Upstream Prioritized Switching and Earliest Arrival Prioritized Switching.

\subsection{Basic performance}

Fig. 4 shows throughput performance. The throughput is defined as the amount of receiving data to that of arrival data. We evaluate the performance of UPS and EAPS respectively about the case both with and without retransmission. It is shown that throughput of all deteriorates from the middle. This is because OBS has many channel collision due to the one-way signaling.

Fig. 5 shows goodput performance. The goodput is defined as the amount of receiving data to that of transmitting data. In the case of UPS, goodput performance deteriorates due to the transmit interruption. On the other hand, 


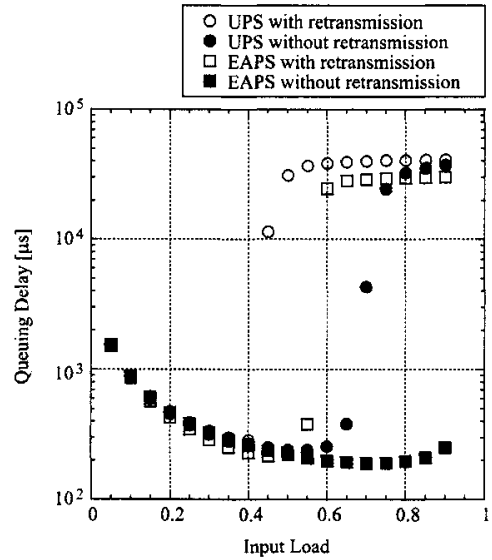

Figure 6. Comparison of UPS and EAPS about average queueing delay

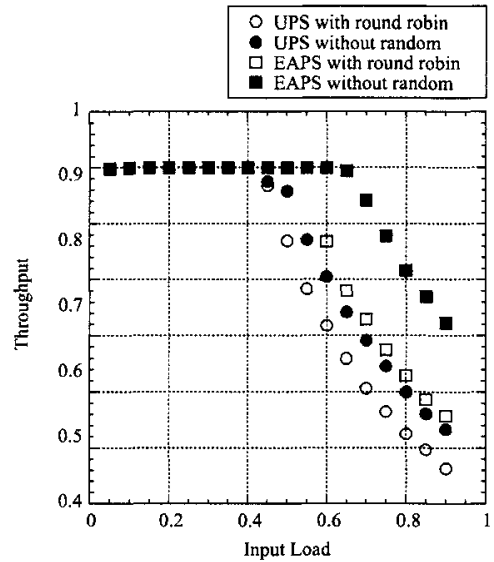

Figure 7. Effect of random queue selection to throuput performance

EAPS decrease the goodput by discarding burst at intermediate nodes. It is shown that UPS maintains higher goodput than EAPS.

Fig. 6 shows average queueing delay. Queueing delay is defined as the time interval from the instance that the packet arrives at a node to the instance that the packet leaves the node. We observe that, as the average arrival rate increases, the Queueing delay first decreases, and then it increases. This is due to the fact that when the traffic intensity is low, the time for a transmit queue to reach the Min Burst Size. Since many collisions occur under a high load, queueing delay becomes very long (but the maximum delay is restricted by buffer size).

\subsection{Effect of random queue selection}

We evaluate the performance of UPS and EAPS with retransmission about the case both round-robin queue selection and random queue selection. From Fig. 7, we notice that throughput performance of both UPS and EAPS are improved by appling proposed random queue selection. Especially, it is effictive to EAPS. Moreover, the tendency is the same in Fig. 8. On the other hand, we notice that an effect to delay performance is slightly, as shown in Fig. 9.

\section{CONCLUSION}

In this paper, we have proposed an architecture of Optical Burst Switched WDM ring network. In our proposed OBS ring network, every node is equipped with one tunable transmitter and one fixed-tuned receiver (TTFR) oparating on a given wavelength that identifies the node. TTFR type ring network has an 


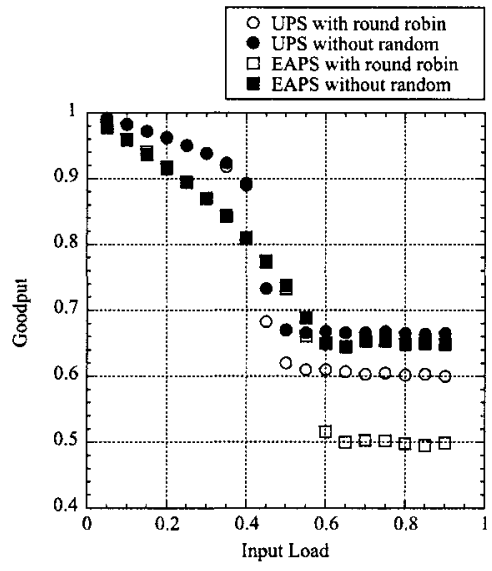

Figure 8. Effect of random queue selection to goodput performance

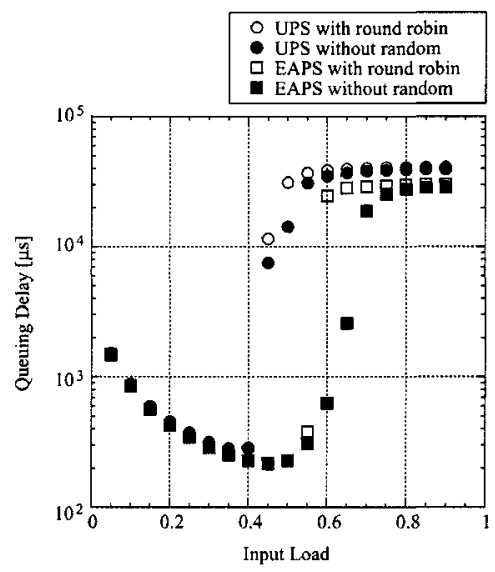

Figure 9. Effect of random queue selection to average queueing delay

advantage that no receiver collisions occur. By computer simulations, we evaluate the performance of throughput, goodput, and queueing delay. As a result, we show the OBS ring network achieves good performance under a low load. It is shown that our proposed random queue selection can improve the performance at a high load.

\section{ACKNOWLEDGMENTS}

This work is partly supported by Keio University COE Program in "Optical and Electronic Device on Access Network" by Ministry of Education, Culture, Sports, Science and Technology, Japan and Support Center for Advanced Telecommunications Technology Research, Foundation.

\section{REFERENCES}

[1] C.Qiao and M.Yoo, "Optical burst switching(OBS) - A new paradigm for an optical internet,"J. High Speed Networks, vol.8, pp.69-84,1999.

[2] Yijun Xiong, Marc Vandenhoute and Hakki C. Cankaya, "Control Archtecutre in Optical Burst-Swtiched WDM Networks,"IEEE Journal on Selected Areas in Communications, vol.18, no.10, pp.1838-1851, Oct. 2000.

[3] K. Shrikhande, I. M. White, D. Wonglumsom, S. M. Gemelos, M. S. Rogge, Y. Fukashiro, M. Avenarius and L. G. Kazovsky, "HORNET: A Packet-Over-WDM Multiple Access Metropolitan Area Ring Network,"IEEE Journal on Selected Areas in Communications, vol.18, no.10, pp. 2004-2016, Oct. 2000.

[4] Lisong $\mathrm{Xu}$, Harry G. Perros, George N. Rouskas, "A simulation study of optical burst switching and access protocols for WDM ring networks,"Computer Networks, 41, pp.143$160,2003$.

[5] W. Fischer, K. Meier-Hellstern, "The Markov-modulated Poisson process (MMPP) cookbook,"Performance Evaluation, 18, pp.149-171, 1992. 